\title{
Surface differentiation by parametric modeling of infrared intensity scans
}

\author{
Tayfun Aytaç \\ Billur Barshan \\ Bilkent University \\ Department of Electrical Engineering \\ Bilkent, TR-06800 \\ Ankara, Turkey \\ E-mail: billur@ee.bilkent.edu.tr
}

\begin{abstract}
We differentiate surfaces with different properties with simple low-cost IR emitters and detectors in a location-invariant manner. The intensity readings obtained with such sensors are highly dependent on the location and properties of the surface, which complicates the differentiation and localization process. Our approach, which models IR intensity scans parametrically, can distinguish different surfaces independent of their positions. Once the surface type is identified, its position $(r, \theta)$ can also be estimated. The method is verified experimentally with wood; Styrofoam packaging material; white painted matte wall; white and black cloth; and white, brown, and violet paper. A correct differentiation rate of $100 \%$ is achieved for six surfaces, and the surfaces are localized within absolute range and azimuth errors of $0.2 \mathrm{~cm}$ and $1.1 \mathrm{deg}$, respectively. The differentiation rate decreases to $86 \%$ for seven surfaces and to $73 \%$ for eight surfaces. The method demonstrated shows that simple IR sensors, when coupled with appropriate signal processing, can be used to recognize different types of surfaces in a location-invariant manner. (c) 2005 Society of Photo-Optical Instrumentation Engineers. [DOI: 10.1117/1.1931467]
\end{abstract}

Subject terms: surface differentiation; infrared sensors; position estimation; Lambertian reflection; Phong model; pattern recognition; feature extraction; optical sensing.

Paper 040716R received Sep. 28, 2004; revised manuscript received Dec. 25, 2004; accepted for publication Jan. 9, 2004; published online Jun. 20, 2005.

\section{Introduction}

Surface recognition and localization is of considerable interest for intelligent autonomous systems that must explore their environment and identify different types of surfaces in a cost-effective manner. In this paper, we propose the use of a simple IR sensor consisting of one emitter and one detector, where the emitted light is reflected from the target and the return intensity is measured at the detector. Although these devices are inexpensive, practical, and widely available, their use has been mostly limited to the detection of the presence or absence of objects in the environment for applications such as obstacle avoidance or counting. Gathering further information about the objects with simple IR sensors has not been much investigated. However, due to the limited resources of autonomous systems, the available resources must be exploited as much as possible. This means that the ability of simple sensor systems to extract information about the environment should first be maximally exploited before more expensive sensing modalities with higher resolution and higher resource requirements (such as computing power) are considered for a given task. Therefore, one of the aims of this study is to explore the limits of simple and inexpensive IR sensors for surface recognition and localization to extend their usage to tasks beyond simple proximity detection.

One problem with the use of simple IR detectors is that it is not possible to deduce the surface properties and the geometry of the reflecting target based on a single intensity return without knowing its position and orientation, be-

0091-3286/2005/\$22.00 @ 2005 SPIE cause the reflected light depends highly on the distance and the angular orientation of the reflecting target. Similarly, one cannot make accurate range estimates based on a single intensity return. Due to single intensity readings not providing much information about an object's properties, the recognition capabilities of IR sensors have been underestimated and underused in most work. One way around this problem is to employ IR sensors in combination with other sensing modalities to acquire information about the surface properties of the object once its distance is estimated. Such an approach is taken in Refs. 1 and 2, where colors are differentiated by employing IR and ultrasonic sensors in a complementary fashion. Reference 3 is based on a similar approach, where the properties of planar surfaces at a known distance (measured by an ultrasonic sensor) are determined first. Once the surface type is determined, the IR sensor is used as a range finder for the same type of surface at other distances. In this paper, we propose a scanning technique to collect intensity signals and a method for surface recognition by parametric modeling of IR intensity scans. The proposed approach can differentiate a moderate number of surfaces and estimate their positions accurately. Our results indicate that if the data acquired from such simple IR sensors are processed effectively through the use of suitable techniques, substantially more information about the environment can be extracted with these devices than in their typical applications.

The use of IR sensing in the pattern recognition area has been mostly limited to the recognition or detection of features or targets in conventional 2-D images. Examples of work in this category include face identification, ${ }^{4}$ automatic vehicle detection, ${ }^{5}$ automatic target recognition $^{6}$ and 
tracking, ${ }^{7}$ detection and identification of targets in background clutter, ${ }^{8}$ remote sensing, and automated terrain analysis. ${ }^{9}$

IR sensors are used in robotics and automation, process control, remote sensing, and safety and security systems. More specifically, they have been used in simple object and proximity detection, ${ }^{10}$ counting, ${ }^{11}$ distance and depth monitoring, floor sensing, position measurement and control, ${ }^{12}$ obstacle and collision avoidance, ${ }^{13}$ and map building. ${ }^{14}$ IR sensors are used in door detection and mapping of openings in walls, ${ }^{15}$ as well as monitoring doors and windows of buildings and vehicles, and "light curtains" for protecting an area. References 16 and 17 deal with the optical determination of depth information. Reference 18 describes a passive IR sensing system that identifies the locations of the people in a room. IR sensors have also been used for automated sorting of waste objects made of different materials. $^{19}$

In our earlier works, ${ }^{20-22}$ we considered the differentiation and localization of objects using a template-based approach, which uses distinctive natures of the IR intensity scans. In Ref. 20, a correct classification rate of $97 \%$ was achieved with absolute range and azimuth errors of $0.8 \mathrm{~cm}$ and $1.6 \mathrm{deg}$ for targets with different geometrical properties, but made of the same surface material (unpolished wood). A rule-based approach to the same problem can be found in Ref. 23, where we achieve an average correct target differentiation rate of $91.3 \%$ over four target types with average absolute range and azimuth errors of $0.55 \mathrm{~cm}$ and $1.03 \mathrm{deg}$, respectively. The advantages of a rule-based approach are shorter processing time, minimal storage requirements, and greater robustness to noise and deviations in geometry and surface properties, since the rule-based approach emphasizes structural features rather than the exact functional forms of the scans. In Ref. 21, targets made of different surface materials but of the same planar geometry are differentiated with a correct differentiation rate of $87 \%$ and absolute range and azimuth errors of $1.2 \mathrm{~cm}$ and $1.0 \mathrm{deg}$. In Ref. 22, we dealt with the problem of differentiating and localizing targets whose geometry and surface properties both vary, generalizing and unifying the results of Refs. 20 and 21. A correct classification rate of $80 \%$ of both geometry and surface over all target types considered is achieved and targets are localized within absolute range and azimuth errors of $1.5 \mathrm{~cm}$ and $1.1 \mathrm{deg}$, respectively. Our approach in these earlier works can be considered as nonparametric, unlike the approach taken in this paper.

This paper is organized as follows. Section 2 reviews some existing reflection models and discusses our parametric modeling of IR intensity scans. Section 3 provides experimental verification of the approach presented in this paper. Concluding remarks are made in the last section.

\section{Modeling of IR Intensity Scans}

Light reflected from a surface depends on the wavelength, the distance, and the properties of the light source (i.e., point or diffuse source), as well as the properties of the surface under consideration such as reflectivity, absorbtivity, transmittivity, and orientation. ${ }^{24}$ Depending on the surface properties, reflectance can be modeled in different ways.
Matte materials can be approximated as ideal Lambertian surfaces, which absorb no light and reflect all the incident light equally in all directions such that the intensity of the reflected light is proportional to the cosine of the angle between the incident light and the surface normal. ${ }^{24-26}$ This is known as Lambert's cosine law. ${ }^{27}$

When a Lambertian surface is illuminated by a point source of radiance $l_{i}$, then the radiance reflected from the surface will be

$l_{\mathrm{s}, L}=l_{\mathrm{i}}\left[k_{d}(\boldsymbol{l}, \boldsymbol{n})\right]$,

where $k_{d}$ is the coefficient of the diffuse reflection for a given material and $\boldsymbol{l}$ and $\boldsymbol{n}$ are the unit vectors representing the directions of the light source and the surface normal, respectively, as shown in Fig. 1(a).

In perfect or specular (mirror-like) reflection, the incident light is reflected in the plane defined by the incident light and the surface normal, making an angle with the surface normal which is equal to the incidence angle $\alpha$ [Fig. 1(b)].

The Phong model, ${ }^{28}$ which is frequently used in computer graphics applications to represent the intensity of energy reflected from a surface, combines the three types of reflection-ambient, diffuse (Lambertian), and specular reflection-in a single formula:

$l_{\mathrm{s}, \text { total }}=l_{\mathrm{a}} k_{a}+l_{\mathrm{i}}\left[k_{d}(\boldsymbol{l}, \boldsymbol{n})\right]+l_{\mathrm{i}}\left[k_{s}(\boldsymbol{r}, \boldsymbol{v})^{m}\right]$,

where $l_{\mathrm{s} \text {,total }}$ is the total radiance reflected from the surface; $l_{\mathrm{a}}$ and $l_{\mathrm{i}}$ are the ambient and incident radiances on the surface; $k_{a}, k_{d}$, and $k_{s}$ are the coefficients of ambient light and diffuse and specular reflection for a given material; $\boldsymbol{l}, \boldsymbol{n}$, $\boldsymbol{r}$, and $\boldsymbol{v}$ are the unit vectors representing the directions of the light source, the surface normal, the reflected light, and the viewing angle, respectively, as shown in Fig. 1(b), and $m$ refers to the order of the specular fall-off or shine. The scalar product in the second term of the Phong model equals $\cos \alpha$, where $\alpha$ is the angle between the vectors $l$ and $\boldsymbol{n}$. Similarly, the scalar product in the last term of the Phong model equals $\cos \beta$, where $\beta$ is the angle between $\boldsymbol{r}$ and $\boldsymbol{v}$. Since the IR emitter and receiver are situated at approximately the same position, then the angle $\beta$ between the reflected vector $\boldsymbol{r}$ and the viewing vector $\boldsymbol{v}$ is equal to $2 \alpha$.

In Ref. 3, the simple nonempirical mathematical model represented by Eq. (2) is used to model reflections from planar surfaces located at a known distance $(10 \mathrm{~cm})$ by fitting the reflectance data to the model to improve the accuracy of the range estimates of IR sensors over a limited range interval (5 to $23 \mathrm{~cm}$ ). A similar approach with a simplified reflection model is employed in Ref. 29, where an IR-sensor-based system can measure distances up to $1 \mathrm{~m}$. The requirement of prior knowledge of the distance to the surface is eliminated in Refs. 30 and 31 by considering two angular intensity scans taken at two different known distances $(10$ and $12 \mathrm{~cm})$. The distance error is less than $1 \mathrm{~cm}$ over a very limited range interval $(10$ to $18 \mathrm{~cm})$ for the reflection coefficients found based on the scans at 10 and $12 \mathrm{~cm}$. As the distance increases to the maximum operating range $(24 \mathrm{~cm})$, the distance error increases, as reported in Refs. 30 and 31. For five different surfaces, a correct classification rate of $75 \%$ is achieved ${ }^{31}$ by considering the in- 


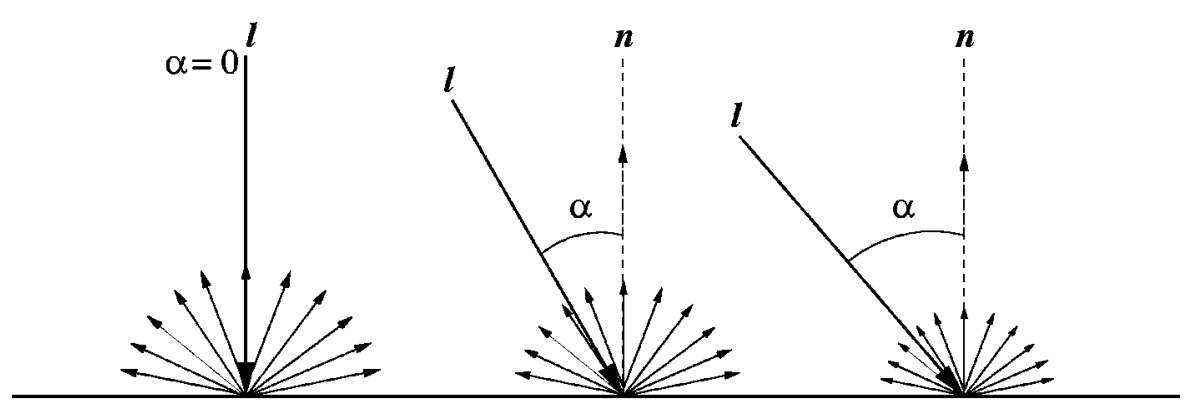

(a)

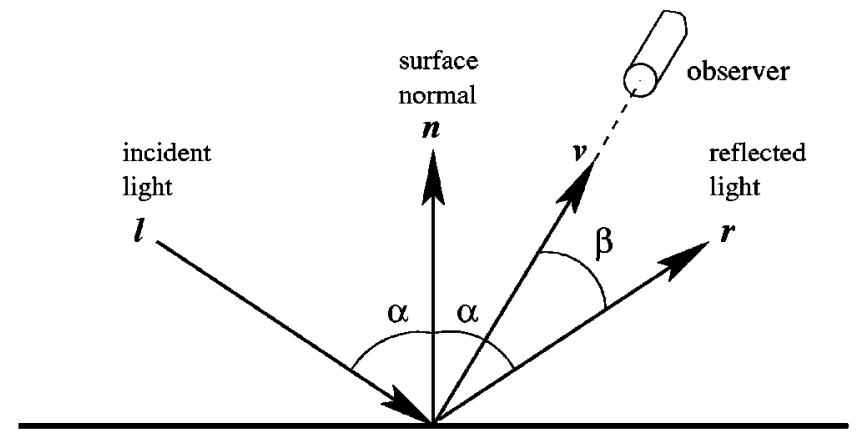

(b)

Fig. 1 (a) Diffuse reflection and (b) specular reflection from an opaque surface.

variance property of the sum of the reflection coefficients below a certain range $(14 \mathrm{~cm})$. In the same study, the authors alternatively propose to use the maximum intensity values at a known range for improved surface differentiation, which requires prior knowledge or estimation of the range to the surface. In Ref. 32, the recognition capabilities of active infrared sensor arrays are analyzed by simulation of infrared signal propagation, using the model represented by Eq. (2).

Our approach differs from those in Refs. 3 and 29 in that it takes distance as a variable and does not require prior knowledge of the distance. Another difference is that those works concentrate mainly on range estimation over a very limited range interval rather than the determination of the surface type, whereas in this paper, we focus on the determination of the surface type over a broader range interval. When we compare our results with those of Refs. 30 and 31 , we can conclude that the proposed approach is better in terms of the correct differentiation rate and the number of surfaces recognized. Furthermore, in the work presented in this paper, we can simultaneously recognize surfaces and estimate their ranges by relating maximum intensity values to the reflection coefficients in a novel way. We also note that the position-invariant pattern recognition and position estimation achieved in this paper is different from such operations performed on conventional images ${ }^{33}$ in that here we work not on direct "photographic" images of the surfaces obtained by some kind of imaging system, but rather on intensity scans obtained by rotating a point sensor. As such, position-invariant differentiation and localization is achieved with an approach quite different from those employed in invariant pattern recognition and localization in conventional images. ${ }^{34}$

The surface materials considered are unpolished wood;
Styrofoam packaging material; white painted matte wall; white and black cloth; and white, brown, and violet paper (not glossy). The IR sensor ${ }^{35}$ [see Fig. 2(a)] is mounted on a 12 -in. rotary table ${ }^{36}$ to obtain angular intensity scans from these surfaces. A photograph of the experimental setup and its schematic can be seen in Figs. 2(b) and 3, respectively.

Reference intensity scans were collected for each surface type by locating the surfaces between 30 and $52.5 \mathrm{~cm}$ with $2.5-\mathrm{cm}$ distance increments at $\theta=0 \mathrm{deg}$. The resulting reference scans for the eight surfaces are shown in Fig. 4 using dotted lines. These intensity scans were modeled by approximating the surfaces as ideal Lambertian surfaces since all of the surface materials involved had matte surfaces. The received return signal intensity is proportional to the detector area and inversely proportional to the square of the distance to the surface and is modeled with three parameters as

$\mathcal{I}=\frac{C_{0} \cos \left(\alpha C_{1}\right)}{[z / \cos \alpha+R(1 / \cos \alpha-1)]^{2}}$,

which is a modified version of the second term in the model represented by Eq. (2). In our case, the ambient reflection component, which corresponds to the first term in Eq. (2), can be neglected with respect to the other terms because the IR filter, covering the detector window, filters out this term. Furthermore, the second term in Eq. (2), representing Lambertian reflection, dominates the third term for the matte surface types considered in this study, as further discussed in the following paragraph. In Eq. (3), the product of the intensity of the emitter, the area of the detector, and the reflection coefficient of the surface are lumped into the constant $C_{0}$, and $C_{1}$ is an additional coefficient to compensate 


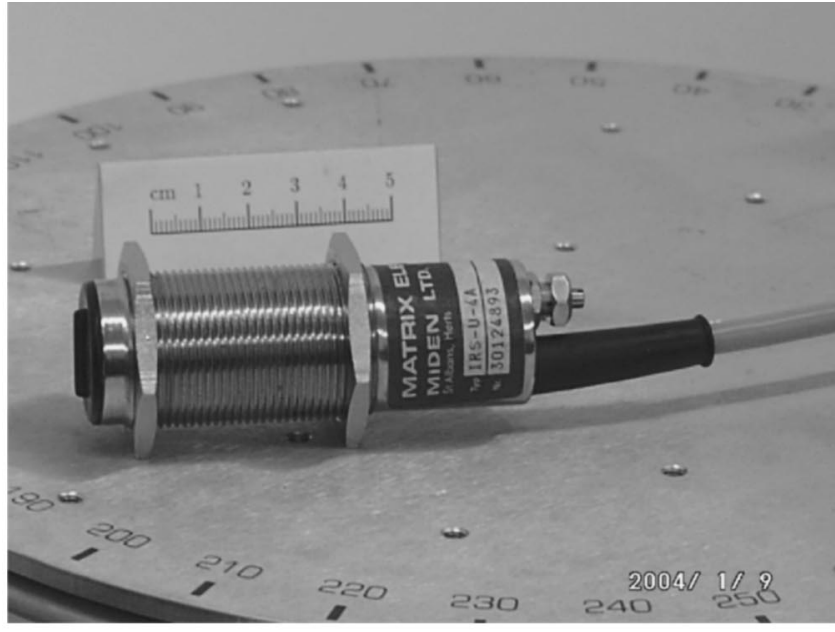

(a)

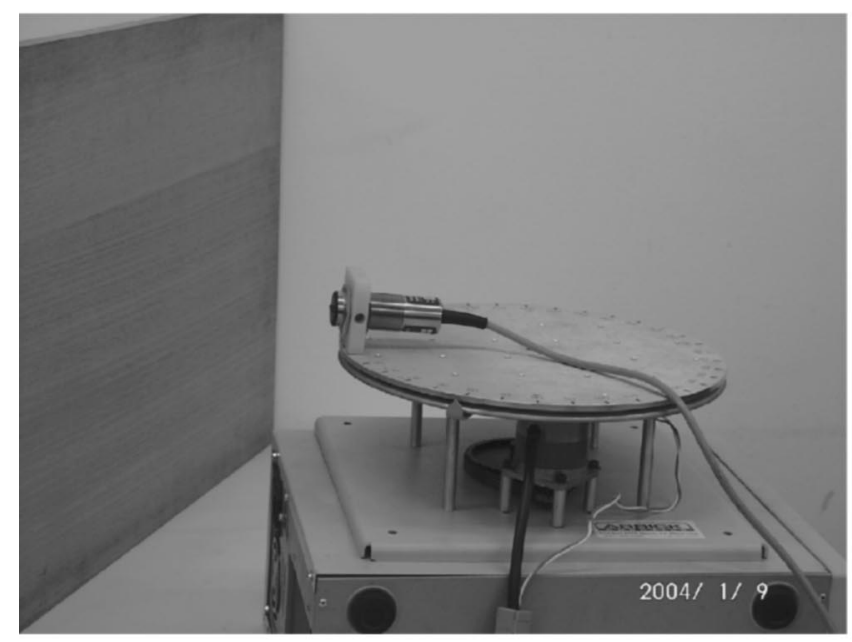

(b)

Fig. 2 (a) The IR sensor and (b) the experimental setup.

for the change in the basewidth of the intensity scans with respect to distance (Fig. 4). A similar dependence on $C_{1}$ is used in sensor modeling in Ref. 37. The $z$ is the horizontal distance between the rotary platform and the surface, as shown in Fig. 3. The denominator of $\mathcal{I}$ is the square of the distance $d$ between the IR sensor and the surface. From the geometry of Fig. 3, $d+R=(z+R) / \cos \alpha$, from which we obtain $d$ as $z / \cos \alpha+R(1 / \cos \alpha-1)$, where $R$ is the radius of the rotary platform and $\alpha$ is the angle made between the IR sensor and the horizontal.

Besides the model represented by Eq. (3), we checked the suitability of a number of other models to our experimental data, which were basically different variations of Eq. (2). The increase in the number of model parameters results in overfitting to the experimental data, whereas simpler models result in larger curve fitting errors. The model represented by Eq. (3) was the most suitable in the sense that it provided a reasonable trade-off.

Using the model represented by Eq. (3), parameterized curves were fitted to the reference intensity scans employing a nonlinear least-squares technique based on a modeltrust region method provided ${ }^{38}$ by MATLAB ${ }^{\mathrm{TM}}$. The result-

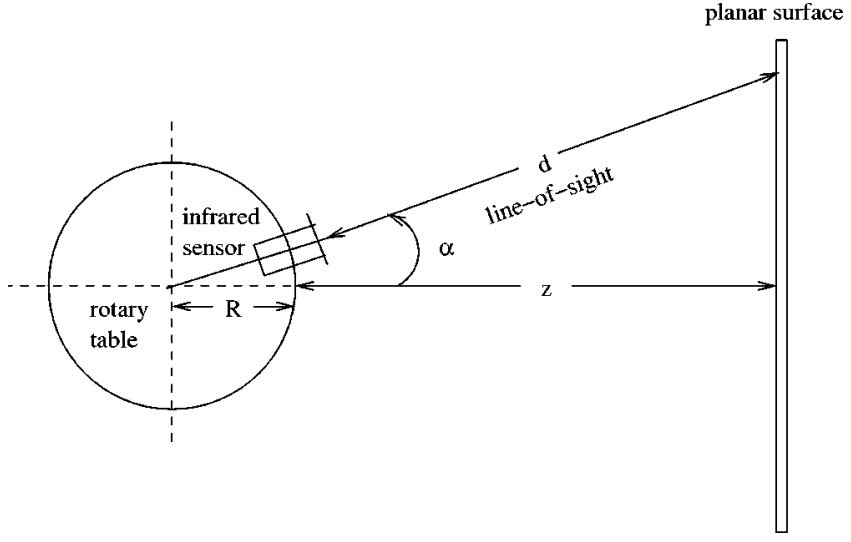

Fig. 3 Top view of the experimental setup used in surface recognition and localization. The emitter and detector windows are circular with 8-mm diameter and center-to-center separation of $12 \mathrm{~mm}$. (The emitter is above the detector.) Both the scan angle $\alpha$ and the surface azimuth $\theta$ are measured counterclockwise from the horizontal axis.

ing curves are shown in Fig. 4 as solid lines. For the reference scans, $z$ is not taken as a parameter since the distance between the surface and the IR sensing unit is already known. The initial guesses of the parameters must be made cleverly so that the algorithm does not converge to local minima, and curve fitting is achieved in a smaller number of iterations. The initial guess for $C_{0}$ is made by evaluating $\mathcal{I}$ at $\alpha=0 \mathrm{deg}$, and corresponds to the product of $\mathcal{I}$ with $z^{2}$. Similarly, the initial guess for $C_{1}$ is made by evaluating $C_{1}$ from Eq. (3) at a known angle $\alpha$ other than zero, with the initial guess of $C_{0}$ and the known value of $z$. While curve fitting, the $C_{0}$ value is allowed to vary between \pm 2000 of its initial guess and $C_{1}$ is restricted to be positive. The variations of $C_{0}, C_{1}$, and $z$ with respect to the maximum intensity of the reference scans are shown in Fig. 5. As the distance $d$ decreases, the maximum intensity increases and $C_{0}$ first increases then decreases, but $C_{1}$ and $z$ both decrease, as expected from the model represented by Eq. (3). The model fit is much better for scans with smaller maximum intensities because our model takes only diffuse reflections into account, but the contribution of the specular reflection components around the maximum value of the intensity scans increases as the distance decreases. Hence, the operating range of our system is extended at the expense of the error at nearby ranges.

\section{Experimental Verification and Discussion}

In this section, we experimentally verify the proposed method. In the test process, the surfaces are randomly located at azimuth angles varying from -45 to $45 \mathrm{deg}$, and range values between 30 to $52.5 \mathrm{~cm}$. In the given region, the return signal intensities do not saturate. In fact, we experimented with fitting models to the saturated scans so that the operating range of the system is extended to include the saturation regions. However, these trials were not very successful. For unsaturated scans, first, the maximum intensity of the observed intensity scan is found and the angular value where this maximum occurs is taken as the azimuth estimate of the surface. If there are multiple maximum intensity values, the average of the minimum and maximum 


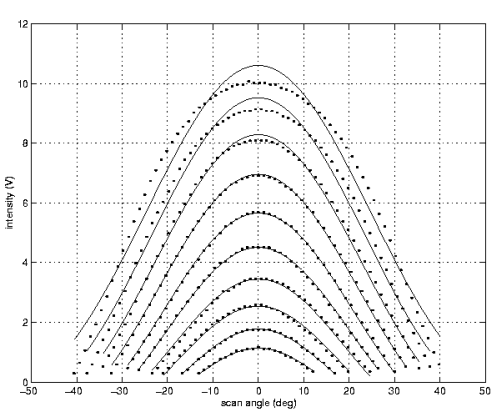

(a)

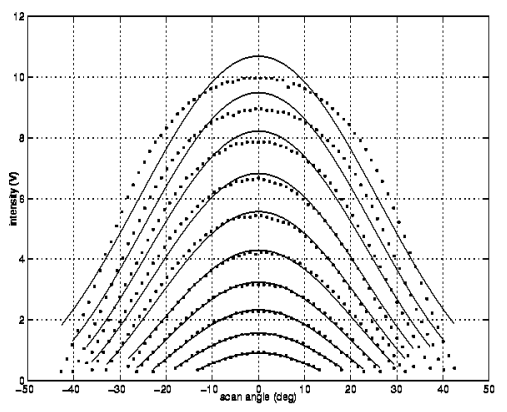

(d)

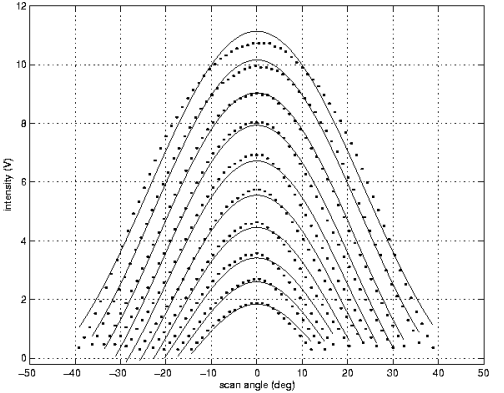

(b)

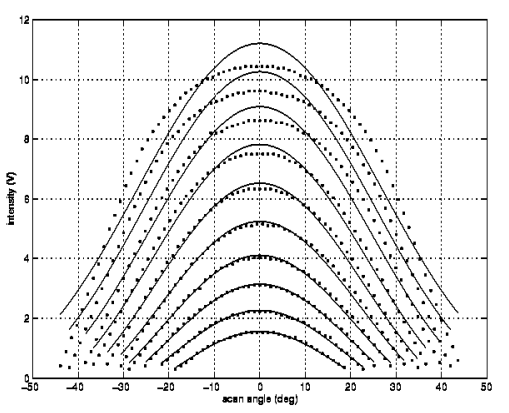

(e)

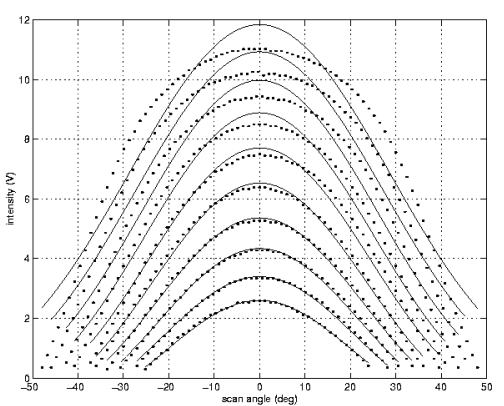

(c)

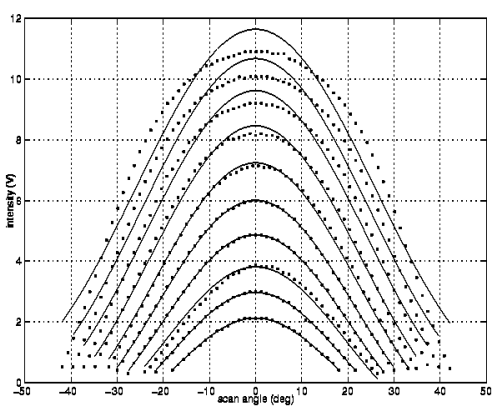

(f)

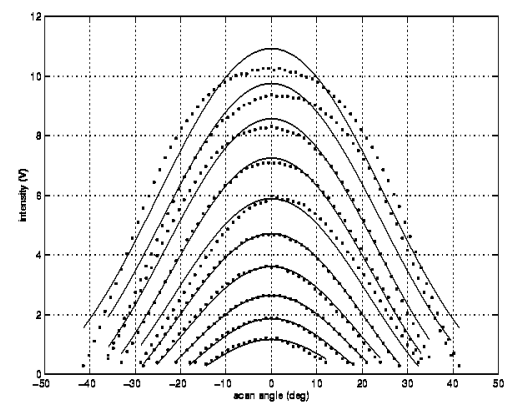

(g)

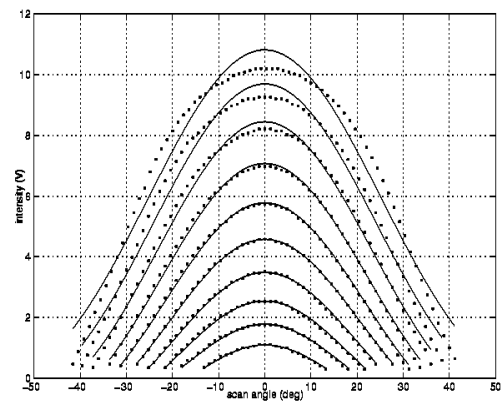

(h)

Fig. 4 Intensity scans of the eight surfaces collected between 30 and $52.5 \mathrm{~cm}$ in $2.5-\mathrm{cm}$ increments. Solid lines indicate the model fit and the dotted lines indicate the experimental data for (a) wood, (b) Styrofoam, (c) white painted matte wall, (d) white cloth, (e) black cloth, (f) white paper, (g) brown paper, and $(\mathrm{h})$ violet paper.

angular values where the maximum intensity values occur is calculated to find the azimuth estimate of the surface. Then, the observed scan is shifted by the azimuth estimate and the model represented by Eq. (3) is fitted using a model-trust region based nonlinear least-squares technique. ${ }^{38}$ The initial guess for the distance $z$ is found from Fig. 5(c) by taking the average of the maximum possible and the minimum possible range values corresponding to the maximum value of the recorded intensity scan. (Linear interpolation is used between the data points in the figure.) This results in a maximum absolute range error of approximately $2.5 \mathrm{~cm}$. Therefore, the parameter $z$ is allowed to vary between $\pm 2.5 \mathrm{~cm}$ of its initial guess. Using the initial guess for $z$, the initial guesses for $C_{0}$ and $C_{1}$ are made in the same way as already explained for the reference scans. After nonlinear curve fitting to the observed scan, we obtain three parameters $C_{0}^{*}, C_{1}^{*}$, and $z^{*}$. In the decision process, the maximum intensity of the observed scan is used, and a value of $C_{1}$ is obtained by linear interpolation between the data points in Fig. 5(b) for each surface type. In other words, Fig. 5(b) is used like a look-up table. Surface-type decisions are made based on the absolute difference of $C_{1}-C_{1}^{*}$ for each surface because of the more distinctive nature of the $C_{1}$ variation with respect to the maximum intensity. The surface type giving the minimum difference is chosen as the correct one. The decision could have also been made by comparing the parameters with those at the estimated range. However, this would not give better results because of the error and the uncertainty in the range estimates. We also considered taking different combinations of the differences $C_{0}-C_{0}^{*}, C_{1}-C_{1}^{*}$, and $z$ $-z^{*}$ as our error criterion. However, the criterion based on $C_{1}-C_{1}^{*}$ difference was the most successful. 


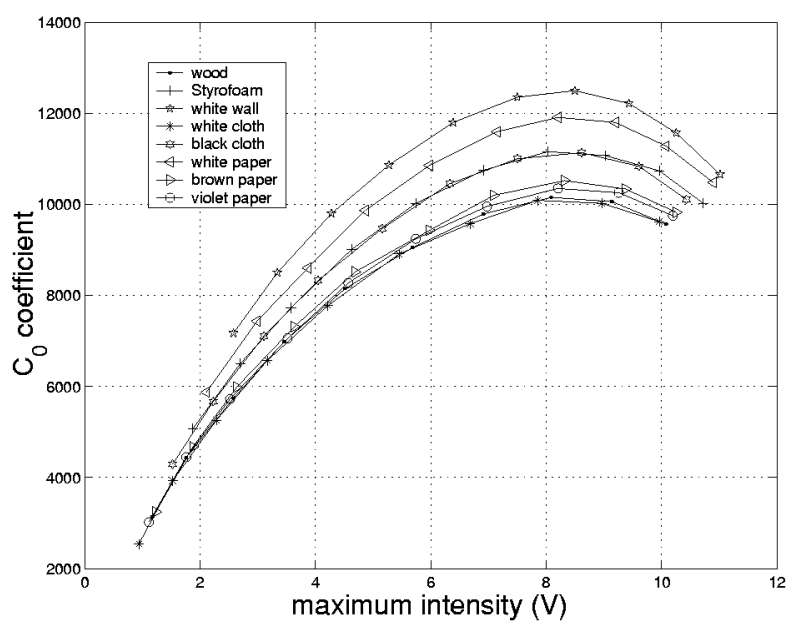

(a)

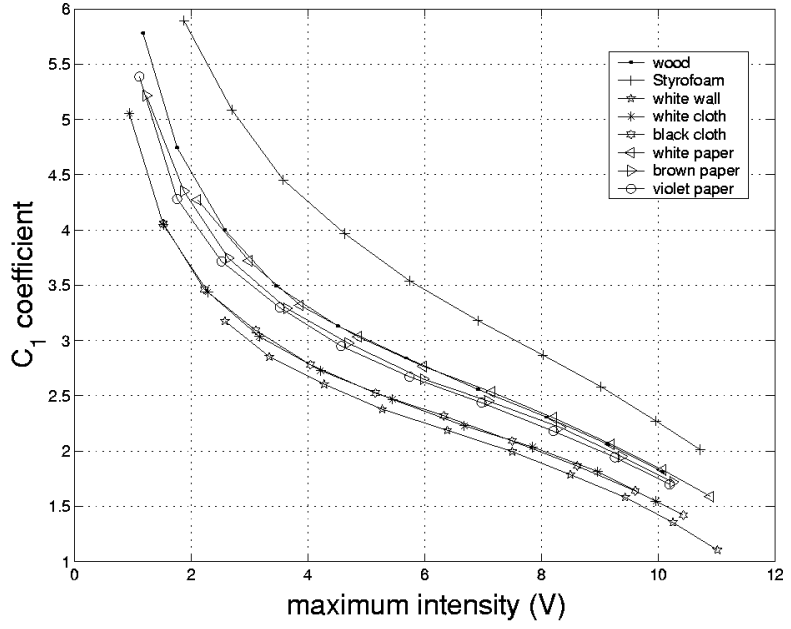

(b)

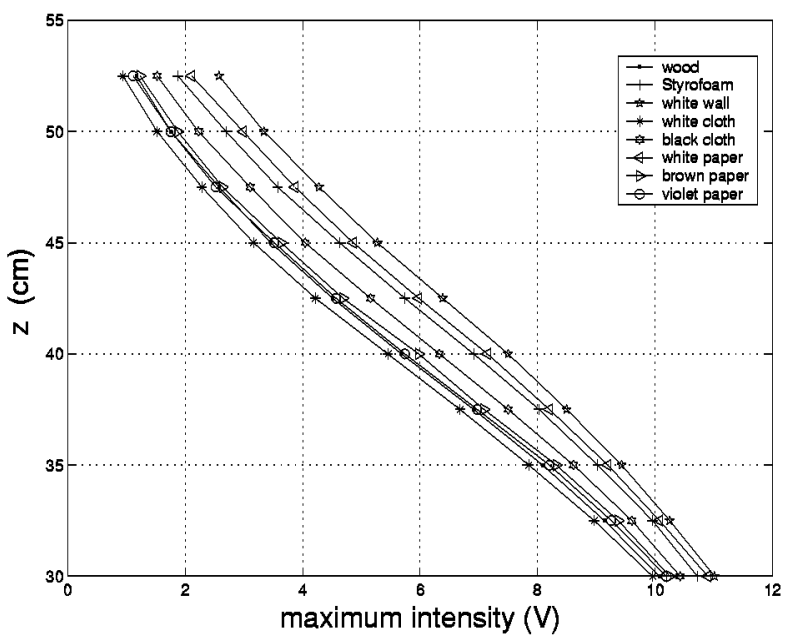

(c)

Fig. 5 Variations of the parameters (a) $C_{0}$, (b) $C_{1}$, and (c) $z$ with respect to maximum intensity of the scan.

For a set of six surfaces including Styrofoam packaging material; white painted matte wall; white or black cloth; and white, brown, and violet paper (also matte), we get a correct differentiation rate of $100 \%$ and the surfaces are located with absolute range and azimuth errors of $0.2 \mathrm{~cm}$ and $1.1 \mathrm{deg}$, respectively. We can increase the number of surfaces differentiated at the expense of a decrease in the correct differentiation rate. For example, if we add wood to our test set and keep either white or black cloth, we get a correct differentiation rate of $86 \%$ for seven surfaces (Table 1). For these sets of surfaces, absolute range and azimuth errors are $0.6 \mathrm{~cm}$ and $1.1 \mathrm{deg}$, respectively. Similarly, if we form a set of surfaces excluding wood but keeping both white and black cloth, we achieve a correct differentiation rate of $83 \%$ for seven surfaces (Table 2) and the surfaces are located with absolute range and azimuth errors of 0.5 $\mathrm{cm}$ and $1.1 \mathrm{deg}$, respectively. The recognition results for all eight surfaces considered are tabulated in Table 3. Over these eight surfaces, an overall correct differentiation rate of $73 \%$ is achieved and surfaces are located with absolute
Table 1 Surface confusion matrix: $C_{1}$-based differentiation (initial range to the surface is estimated using the maximum intensity of the scan).

\begin{tabular}{lcccccccc}
\hline \hline & \multicolumn{7}{c}{ Differentiation Results } \\
\cline { 2 - 7 } Surface & WO & ST & WW & WC(BC) & WP & BR & VI & Total \\
\hline WO & 4 & - & - & - & 7 & - & 1 & 12 \\
ST & - & 12 & - & - & - & - & - & 12 \\
WW & - & - & 12 & - & - & - & - & 12 \\
WC(BC) & - & - & - & 12 & - & - & - & 12 \\
WP & 4 & - & - & - & 8 & - & - & 12 \\
BR & - & - & - & - & - & 12 & - & 12 \\
VI & - & - & - & - & - & - & 12 & 12 \\
Total & 8 & 12 & 12 & 12 & 15 & 12 & 13 & 84 \\
\hline \hline WO: & & & & & & &
\end{tabular}

WO: wood, ST: Styrofoam, WW: white painted matte wall, WC: white cloth, BC: black cloth, WP: white paper, BR: brown paper, VI: violet paper. 
Table 2 Surface confusion matrix: $C_{1}$-based differentiation (initial range to the surface is estimated using the maximum intensity of the scan).

\begin{tabular}{lcccccccc}
\hline \hline & \multicolumn{7}{c}{ Differentiation Results } \\
\cline { 2 - 7 } Surface & ST & WW & WC & BC & WP & BR & VI & Total \\
\hline ST & 12 & - & - & - & - & - & - & 12 \\
WW & - & 12 & - & - & - & - & - & 12 \\
WC & - & - & 7 & 5 & - & - & - & 12 \\
BC & - & - & 9 & 3 & - & - & - & 12 \\
WP & - & - & - & - & 12 & - & - & 12 \\
BR & - & - & - & - & - & 12 & - & 12 \\
VI & - & - & - & - & - & - & 12 & 12 \\
Total & 12 & 12 & 16 & 8 & 12 & 12 & 12 & 84 \\
\hline \hline
\end{tabular}

range and azimuth errors of $0.8 \mathrm{~cm}$ and $1.1 \mathrm{deg}$, respectively. Referring to Tables 1 to 3 , note that the range estimation accuracy improves with increasing correct classification rate, whereas the azimuth estimation accuracy is independent of it, as expected, because of the way it is estimated. In these tables, white and black cloth as well as wood and white paper are the surface pairs most often confused with each other. Thus, the decrease in the differentiation rate resulting from adding new surfaces does not represent an overall degradation in differentiation rates across all surface types but is almost totally explained by pairwise confusion of the newly introduced surface with a previously existing one, resulting from the similarity of the $C_{1}$ parameter of the intensity scans of the two confused surfaces.

To investigate the effect of the initial range estimate of the surface on the differentiation process, we now assume that the distance to the surface is known beforehand. For this case, only the two variables $C_{0}$ and $C_{1}$ are taken as parameters. Since the azimuth estimation process is independent of range estimation, for the same set of surfaces, the same azimuth estimation results are obtained. Therefore, they are not repeated here. For the same six surfaces considered as in the previous case (where the initial range to the surface is estimated using the maximum intensity of

Table 3 Surface confusion matrix: $C_{1}$-based differentiation (initial range to the surface is estimated using the maximum intensity of the scan).

\begin{tabular}{lccccccccc}
\hline \hline & \multicolumn{7}{c}{ Differentiation Results } \\
\cline { 2 - 9 } Surface & WO & ST & WW & WC & BC & WP & BR & VI & Total \\
\hline WO & 4 & - & - & - & - & 7 & - & 1 & 12 \\
ST & - & 12 & - & - & - & - & - & - & 12 \\
WW & - & - & 12 & - & - & - & - & - & 12 \\
WC & - & - & - & 7 & 5 & - & - & - & 12 \\
BC & - & - & - & 9 & 3 & - & - & - & 12 \\
WP & 4 & - & - & - & - & 8 & - & - & 12 \\
BR & - & - & - & - & - & - & 12 & - & 12 \\
VI & - & - & - & - & - & - & - & 12 & 12 \\
Total & 8 & 12 & 12 & 16 & 8 & 15 & 12 & 13 & 96 \\
\hline \hline
\end{tabular}

Table 4 Surface confusion matrix: $C_{1}$-based differentiation (range to the surface is known).

\begin{tabular}{lcccccccc}
\hline \hline & \multicolumn{7}{c}{ Differentiation Results } \\
\cline { 2 - 7 } Surface & WO & ST & WW & WC(BC) & WP & BR & VI & Total \\
\hline WO & 5 & - & - & - & 6 & - & 1 & 12 \\
ST & - & 12 & - & - & - & - & - & 12 \\
WW & - & - & 12 & - & - & - & - & 12 \\
WC(BC) & - & - & - & 12 & - & - & - & 12 \\
WP & 4 & - & - & - & 8 & - & - & 12 \\
BR & - & - & - & - & - & 12 & - & 12 \\
VI & - & - & - & - & - & - & 12 & 12 \\
Total & 9 & 12 & 12 & 12 & 14 & 12 & 13 & 84 \\
\hline \hline
\end{tabular}

the scan), the same correct classification rate of $100 \%$ is achieved. If we add wood to our test set and keep either white or black cloth, we get a correct differentiation rate of $87 \%$ for seven surfaces (Table 4). Similarly, if we form a set of surfaces excluding wood but keeping both white and black cloth, we achieve a correct differentiation rate of $88 \%$ for seven surfaces (Table 5). The differentiation results over all eight surfaces are given in Table 6, corresponding to a correct differentiation rate of $78 \%$. When we compare these results with those obtained without exact knowledge of the distance to the surface, we can conclude that similar surfaces are confused with each other (wood/white paper and white/black cloth) with smaller confusion rates.

As an alternative, we take as the initial range estimate, the mid-point of the operating range (30 to $52.5 \mathrm{~cm}$ ), which is $41.25 \mathrm{~cm}$ for all surfaces. An overall correct differentiation rate of $65 \%$ over eight different surfaces is achieved (Table 7), which is worse than the two classification alternatives already considered. The surfaces are located with an absolute range error of $1 \mathrm{~cm}$, which is slightly greater than the absolute range error achieved with the initial range estimate using the maximum intensity of the scan. If we exclude wood and white cloth or wood and black cloth from our test set, we get correct differentiation rates of 93 and $94 \%$ for the remaining six surfaces and the surfaces are located with absolute range errors of 0.3 and $0.4 \mathrm{~cm}$, respectively. As azimuth estimation errors are independent of the applied classification techniques, they are not repeated here. Note that for these sets of surfaces, a correct differentiation rate of $100 \%$ was achieved using the classification approaches already considered. These high differentiation rates show that even for a maximum initial guess error of $11.25 \mathrm{~cm}$ in the range estimates, the proposed approach can recognize a moderate number of surfaces with reasonably good accuracy.

\section{Conclusion}

The main accomplishment of this study is that we achieved position-invariant surface differentiation and localization with simple IR sensors despite the fact that their individual intensity readings are highly dependent on the surface position and properties, and this dependence cannot be represented by a simple analytical relationship. The intensity scan data acquired from a simple low-cost IR emitter and detector pair was processed and modeled. Different param- 
Table 5 Surface confusion matrix: $C_{1}$-based differentiation (range to the surface is known).

\begin{tabular}{lcccccccc}
\hline \hline & \multicolumn{7}{c}{ Differentiation Results } \\
\cline { 2 - 7 } Surface & ST & WW & WC & BC & WP & BR & VI & Total \\
\hline ST & 12 & - & - & - & - & - & - & 12 \\
WW & - & 12 & - & - & - & - & - & 12 \\
WC & - & - & 8 & 4 & - & - & - & 12 \\
BC & - & - & 6 & 6 & - & - & - & 12 \\
WP & - & - & - & - & 12 & - & - & 12 \\
BR & - & - & - & - & - & 12 & - & 12 \\
VI & - & - & - & - & - & - & 12 & 12 \\
Total & 12 & 12 & 14 & 10 & 12 & 12 & 12 & 84 \\
\hline \hline
\end{tabular}

eterized reflection models were considered and evaluated to find the most suitable model fit to our experimental data, which also best represents and classifies the surfaces under consideration. The proposed approach can differentiate six different surfaces with $100 \%$ accuracy. In Ref. 21, where we considered differentiation and localization of surfaces by employing nonparametric approaches, a maximum correct differentiation rate of $87 \%$ over four surfaces was achieved. Comparing this rate with that obtained in this paper, we can conclude that the parametric approach is superior to nonparametric ones, in terms of the accuracy, number of surfaces differentiated, and memory requirements, since the nonparametric approaches we considered require the storage of reference scan signals. By parameterizing the intensity scans and storing only their parameters, we eliminated the need to store complete reference scans. The decrease in the differentiation rate resulting from adding new surfaces in the parametric approach does not represent an overall degradation in differentiation rates across all surface types but is almost totally explained by pairwise confusion of the newly introduced surface with a previously existing one, resulting from the similarity of the $C_{1}$ parameter of the intensity scans of the two confused surfaces. (Similar decreases in differentiation rate with increasing number of surfaces or objects are also observed with nonparametric template-based approaches.) As an improvement, one can consider using differentiation tech-

Table 6 Surface confusion matrix: $C_{1}$-based differentiation (range to the surface is known).

\begin{tabular}{lccccccccc}
\hline \hline & \multicolumn{7}{c}{ Differentiation Results } \\
\cline { 2 - 9 } Surface & WO & ST & WW & WC & BC & WP & BR & VI & Total \\
\hline WO & 5 & - & - & - & - & 6 & - & 1 & 12 \\
ST & - & 12 & - & - & - & - & - & - & 12 \\
WW & - & - & 12 & - & - & - & - & - & 12 \\
WC & - & - & - & 8 & 4 & - & - & - & 12 \\
BC & - & - & - & 6 & 6 & - & - & - & 12 \\
WP & 4 & - & - & - & - & 8 & - & - & 12 \\
BR & - & - & - & - & - & - & 12 & - & 12 \\
VI & - & - & - & - & - & - & - & 12 & 12 \\
Total & 9 & 12 & 12 & 14 & 10 & 14 & 12 & 13 & 96 \\
\hline \hline
\end{tabular}

Table 7 Surface confusion matrix: $C_{1}$-based differentiation (initial range estimate is taken as half of the operating range for all surfaces).

\begin{tabular}{lccccccccc}
\hline \hline & \multicolumn{7}{c}{ Differentiation Results } \\
\cline { 2 - 8 } Surface & WO & ST & WW & WC & BC & WP & BR & VI & Total \\
\hline WO & 2 & - & - & - & - & 9 & - & 1 & 12 \\
ST & - & 12 & - & - & - & - & - & - & 12 \\
WW & - & - & 9 & 1 & 2 & - & - & - & 12 \\
WC & - & - & - & 7 & 5 & - & - & - & 12 \\
BC & - & - & - & 10 & 2 & - & - & - & 12 \\
WP & 4 & - & - & - & - & 7 & 1 & - & 12 \\
BR & 1 & - & - & - & - & - & 11 & - & 12 \\
VI & - & - & - & - & - & - & - & 12 & 12 \\
Total & 7 & 12 & 9 & 18 & 9 & 16 & 12 & 13 & 96 \\
\hline \hline
\end{tabular}

niques or learning and/or clustering algorithms that involve more parameters. One possibility is to take a sequential approach. If the estimated $C_{1}$ parameter of the surface matches more than one surface closely, one can then inspect the other parameters of the surface in sequence. This would be faster than taking all the parameters into account all of the time.

This paper demonstrated that simple IR sensors, when coupled with appropriate processing, can be used to extract substantially more information about the environment than such devices are commonly employed for. We expect this flexibility to significantly extend the range of applications in which such low-cost single-sensor-based systems can be used. Specifically, we expect that it will be possible to go beyond relatively simple tasks such as simple object and proximity detection, counting, distance and depth monitoring, floor sensing, position measurement, and obstacle or collision avoidance, and deal with tasks such as differentiation, classification, recognition, clustering, position estimation, map building, perception of the environment and surroundings, autonomous navigation, and target tracking. The approach presented here would be more useful where selfcorrecting operation is possible due to repeated observations and feedback.

The demonstrated system would find application in intelligent autonomous systems such as mobile robots whose task involves surveying an unknown environment consisting of different surface types. Industrial applications where different materials or surfaces must be identified and separated may also benefit from this approach. Current and future work involves designing a more intelligent system whose operating range is adjustable based on an initial range estimate to the surface. This will eliminate saturation and enable the system to accurately differentiate and localize surfaces over a wider operating range. Another issue we are considering is the extension of the model to include specular reflections from glossy surfaces. We are also working on the recognition of surfaces through the use of artificial neural networks to improve the accuracy. Parametric modeling and representation of intensity scans of different geometries (such as corner, edge, and cylinder) is also being considered to employ the proposed approach in the 
simultaneous determination of the geometry and the surface type of targets.

\section{Acknowledgments}

This research was supported by TÜBITAK under BDP and 197E051 grants. The authors would like to thank the Department of Engineering Science of the University of Oxford for donating the IR sensors.

\section{References}

1. V. Genovese, E. Guglielmelli, A. Mantuano, G. Ratti, A. M. Sabatini, and P. Dario, "Low-cost, redundant proximity sensor system for spatial sensing and color-perception," Electron. Lett. 31(8), 632-633 (1995).

2. A. M. Sabatini, V. Genovese, E. Guglielmelli, A. Mantuano, G. Ratti, and P. Dario, "A low-cost composite sensor array combining ultrasonic and infrared proximity sensors," in Proc. IEEE/RSJ Int. Conf. on Intelligent Robots and Systems, Vol. 3, pp. 120-126, Pittsburgh, PA (1995).

3. P. M. Novotny and N. J. Ferrier, "Using infrared sensors and the Phong illumination model to measure distances," in Proc. IEEE Int Conf. on Robotics and Automation, Vol. 2, pp. 1644-1649, Detroit, MI (1999).

4. P. J. Phillips, "Matching pursuit filters applied to face identification," IEEE Trans. Image Process. 7(8), 1150-1164 (1998).

5. I. Pavlidis, P. Symosek, B. Fritz, M. Bazakos, and N. Papanikolopoulos, "Automatic detection of vehicle occupants: the imaging problem and its solution," Mach. Vision Appl. 11(6), 313-320 (2000).

6. H. Kwon, S. Z. Der, and N. M. Nasrabadi, "Adaptive multisensor target detection using feature-based fusion," Opt. Eng. 41(1), 69-80 (2002).

7. T. Tsao and Z. Q. Wen, "Image-based target tracking through rapid sensor orientation change," Opt. Eng. 41(3), 697-703 (2002).

8. Z. Zalevsky, D. Mendlovic, E. Rivlin, and S. Rotman, "Contrasted statistical processing algorithm for obtaining improved target detection performances in infrared cluttered environment," Opt. Eng. 39(10), 2609-2617 (2000)

9. B. Bhanu, P. Symosek, and S. Das, "Analysis of terrain using multispectral images," Pattern Recogn. 30(2), 197-215 (1997).

10. E. Cheung and V. J. Lumelsky, "Proximity sensing in robot manipulator motion planning: system and implementation issues," IEEE Trans. Rob. Autom. 5(6), 740-751 (1989).

11. A. J. Hand, "Infrared sensor counts insects," Photonics Spectra 32(11), 30-31 (1998).

12. B. Butkiewicz, "Position control system with fuzzy microprocessor AL220," Lect. Notes Comput. Sci. 1226, 74-81 (1997).

13. V. J. Lumelsky and E. Cheung, "Real-time collision avoidance in teleoperated whole-sensitive robot arm manipulators," IEEE Trans. Syst. Man Cybern. 23(1), 194-203 (1993).

14. H.-H. Kim, Y.-S. Ha, and G.-G. Jin, "A study on the environmental map building for a mobile robot using infrared range-finder sensors," in Proc. IEEE/RSJ Int. Conf. on Intelligent Robots and Systems, Vol. 1, pp. 711-716, Las Vegas, NV (2003).

15. A. M. Flynn, "Combining sonar and infrared sensors for mobile robot navigation," Int. J. Robot. Res. 7(6), 5-14 (1988)

16. P. Klysubun, G. Indebetouw, T. Kim, and T. C. Poon, "Accuracy of three-dimensional remote target location using scanning holographic correlation," Opt. Commun. 184(5-6), 357-366 (2000).

17. J. J. Esteve-Taboada, P. Refregier, J. Garcia, and C. Ferreira, "Target localization in the three-dimensional space by wavelength mixing," Opt. Commun. 202(1-3), 69-79 (2002)

18. K. Hashimoto, T. Tsuruta, K. Morinaka, and N. Yoshiike, "High performance human information sensor," Sens. Actuators, A 79(1), 46-52 (2000).

19. D. M. Scott, "A 2-color near-infrared sensor for sorting recycled plastic waste," Meas. Sci. Technol. 6(2), 156-159 (1995).

20. T. Aytaç and B. Barshan, "Differentiation and localization of targets using infrared sensors," Opt. Commun. 210(1-2), 25-35 (2002).

21. B. Barshan and T. Aytaç, "Position-invariant surface recognition and localization using infrared sensors," Opt. Eng. 42(12), 3589-3594 (2003).

22. T. Aytaç and B. Barshan, "Simultaneous extraction of geometry and surface properties of targets using simple infrared sensors," Opt. Eng. 43(10), 2437-2447 (2004)

23. T. Aytaç and B. Barshan, "Rule-based target differentiation and position estimation based on infrared intensity measurements," Opt. Eng. 42(6), 1766-1771 (2003).
24. S. K. Nayar, K. Ikeuchi, and T. Kanade, "Surface reflection: physical and geometrical perspectives," IEEE Trans. Pattern Anal. Mach. Intell. 13(7), 611-634 (1991).

25. M. D. Adams, "Lidar design, use, and calibration concepts for correct environmental detection," IEEE Trans. Rob. Autom. 16(6), 753-761 (2000).

26. E. R. Davies, Machine Vision: Theory, Algorithms, Practicalities, Academic Press, London (1990)

27. M. Born and E. Wolf, Principles of Optics, Pergamon Press, Oxford, UK (1980).

28. B. T. Phong, "Illumination for computer generated pictures," Commun. ACM 18(6), 311-317 (1975).

29. G. Benet, F. Blanes, J. E. Simó, and P. Pérez, "Using infrared sensors for distance measurement in mobile robots," Rob. Auton. Syst. 40(4), 255-266 (2002)

30. M. A. Garcia and A. Solanas, "Automatic distance measurement and material characterization with infrared sensors," in Proc. 8th RoboCup Int. Symp., Lisbon, Portugal (2004).

31. M. A. Garcia and A. Solanas, "Estimation of distance to planar surfaces and type of material with infrared sensors," in Proc. 17th Int Conf. on Pattern Recognition, Vol. 1, pp. 745-748, Cambridge, UK (2004).

32. B. Iske, B. Jäger, and U. Rückert, "A ray-tracing approach for simulating recognition abilities of active infrared sensor arrays," IEEE Sens. J. 4(2), 237-247 (2004)

33. F. T. S. Yu and S. Jutamulia, Eds., Optical Pattern Recognition, Cambridge University Press, Cambridge, UK (1998).

34. S. Roy, H. H. Arsenault, and Y. Sheng, "Shift-, scale-, rotation and pose-invariant object recognition using centroid wedge sampling and a feature space trajectory classifier," J. Mod. Opt. 50(2), 285-297 (2003).

35. Matrix Elektronik, AG, IRS-U-4A Proximity Switch Datasheet, Oberehrendingen, Switzerland (1995).

36. Arrick Robotics, RT-12 Rotary Positioning Table, Hurst, TX (2002), www.robotics.com/rt12.html

37. G. Petryk and M. Buehler, "Dynamic object localization via a proximity sensor network," in Proc. IEEE/SICE/RSJ Int. Conf. on Multisensor Fusion and Integration for Intelligent Systems, pp. 337-341, Washington DC (1996).

38. T. Coleman, M. A. Branch, and A. Grace, MATLAB Optimization Toolbox, User's Guide, The MathWorks, Inc., Natick, MA (1999).

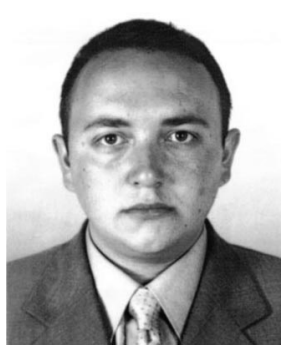

Tayfun Aytaç received his BS degree in electrical engineering in 2000 from Gazi University, Ankara, Turkey, and his MS degree in electrical engineering in 2002 from Bilkent University, Ankara, Turkey, where he is currently working toward his $\mathrm{PhD}$ degree. His current research interests include intelligent sensing, optical sensing, pattern recognition, sensor data fusion, target differentiation, and sensor-based robotics.

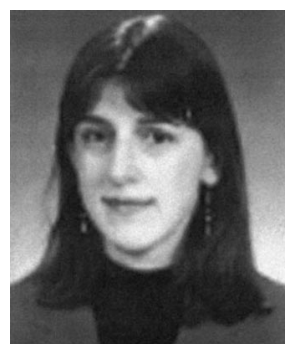

Billur Barshan received her BS degrees in both electrical engineering and physics from Boğaziçi University, Istanbul, Turkey, and her MS and PhD degrees in electrical engineering from Yale University, New $\mathrm{Ha}$ ven, Connecticut, in 1986, 1988, and 1991, respectively. Dr. Barshan was a research assistant with Yale University from 1987 to 1991, and a postdoctoral researcher with the Robotics Research Group at University of Oxford, United Kingdom, from 1991 to 1993. In 1993, she joined Bilkent University, Ankara, where she is currently a professor with the Department of Electrical Engineering, and where she founded the Robotics and Sensing Laboratory. She is the recipient of the 1994 Nakamura Prize awarded to the most outstanding paper at the 1993 IEEE/RSJ Intelligent Robots and Systems International Conference, the 1998 TUBITAK Young Investigator Award, and the 1999 Mustafa N. Parlar Foundation Research Award. Dr. Barshan's current research interests include intelligent sensors, sonar and inertial navigation systems, sensor-based robotics, and multisensor data fusion. 